\title{
Fundamental Mode RF Power Dissipated in a Waveguide Attached to an Accelerating Cavity
}

\author{
Y. W. Kang \\ RF Group \\ Accelerator Systems Division \\ Argonne National Laboratory
}

February 9, 1993

\section{DISCLAIMER}

This report was prepared as an account of work sponsored by an agency of the United States Government. Neither the United States Government nor any agency thereof, nor any of their employees, makes any warranty, express or implied, or assumes any legal liability or responsibility for the accuracy, completeness, or usefulness of any information, apparatus, product, or process disclosed, or represents that its use would not infringe privately owned rights. Reference herein to any specific commercial product, process, or service by trade name, trademark, manufacturer, or otherwise does not necessarily constitute or imply its endorsement, recommendation, or favoring by the United States Government or any agency thereof. The views and opinions of authors expressed herein do not necessarily state or reflect those of the United States Government or any agency thereof. 


\section{Introduction}

An accelerating $R F$ carity usually requires accessory devices such as a tuner, a coupler, and a damper to perform properly. Since a device is attached to the wall of the cavity to have certain electrical coupling of the cavity field through the opening; $R F$ power dissipation is involved. In a high power accelerating cavity, the RF power coupled and dissipated in the opening and in the device must be estimated to design a proper cooling system for the device. The single cell carities of the APS storage ring will use the same accessories. These cavities are rotationally symmetric and the fields around the equator can be approximated with the fields of the cylindrical pillbox cavity. In the following, the coupled and dissipated fundamental mode RF power in a waveguide attached to a pillbox cavity is discussed. The waveguide configurations are 1) aperture- coupled cylindrical waveguide with matched load termination, 2) short- circuited cylindrical waveguide, and 3) E-probe or H-loop coupled coaxial waveguide. A short-circuited, one-wavelength coaxial structure is considered for the fundamental frequency rejection circuit of an H-loop damper. 


\section{Coupled Waveguide Modes}

Figure 1 shows a pillbox cavity with a cylindrical wareguide attached to it. For $T M_{010}$ mode, fields in the cavity are

$$
E_{z}=\frac{k^{2}}{j \omega \epsilon} J_{o}\left(k_{\rho^{\prime}} \rho^{\prime}\right), \quad H_{\phi^{\prime}}=k_{\rho^{\prime}} J_{1}\left(k_{\rho^{\prime}} \rho^{\prime}\right)
$$

where $J_{n}$ is the $n$-th order Bessel function of the first kind. The fundamental mode $\mathrm{RF}$ power dissipated in the cavity with the fields in equation ( 1 ) is

$$
P=\iint_{\text {cavity wall }} R_{s}\left|H_{\phi^{\prime}}\left(\rho^{\prime}, \phi^{\prime}, z\right)\right|^{2} d s
$$

where $R_{s}$ is the surface resistance of the cavity metal

$$
R_{s}=\sqrt{\frac{\omega \mu}{2 \sigma}}
$$

For the cavity input power $P_{i n}$, the fields are

$$
E_{z}=b_{n} \frac{k^{2}}{j \omega \epsilon} J_{o}\left(k_{\rho^{\prime}} \rho^{\prime}\right), \quad H_{\phi^{\prime}}=b_{n} k_{\rho^{\prime}} J_{1}\left(k_{\rho^{\prime}} \rho^{\prime}\right)
$$

where

$$
b_{n}=\sqrt{\frac{P_{\text {in }}}{P}} .
$$

The magnetic field in the aperture $S_{a}$ is assumed to be uniform and $\phi^{\prime}$ - directed.

$$
\hat{\mathbf{a}}_{x} H^{a} \simeq \hat{\mathbf{a}}_{\phi^{\prime}} H_{\phi^{\prime}}
$$

In a circularly cylindrical waveguide, the mode functions are

$$
\begin{aligned}
\mathbf{H}_{n p}^{T M} & =\hat{\mathbf{a}}_{\rho} \frac{j n}{\rho} J_{n}\left(k_{\rho} \rho\right) e^{j n \phi} e^{-j k_{z} z} \\
& -\hat{\mathbf{a}}_{\phi} k_{\rho} J_{n}^{\prime}\left(k_{\rho} \rho\right) e^{j n \phi} e^{-j k_{z} z}
\end{aligned}
$$

for TM modes, where

$$
k_{z}^{2}=\sqrt{k^{2}-k_{\rho}^{2}}, \quad k_{\rho}=\frac{x_{n p}}{a}
$$


and

$$
\begin{aligned}
\mathbf{H}_{n p}^{T E} & =-\hat{\mathbf{a}}_{\rho} \frac{k_{z} k_{\rho}}{\omega \mu} J_{n}^{\prime}\left(k_{\rho} \rho\right) e^{j n \phi} \epsilon^{-j k_{z} z} \\
& -\hat{\mathbf{a}}_{\phi} \frac{j k_{z} n}{\omega \mu \rho} J_{n}\left(k_{\rho} \rho\right) e^{j n \phi} e^{-j k_{z} z} \\
& +\hat{\mathbf{a}}_{z} \frac{k^{2}-k_{z}^{2}}{j \omega \mu} J_{n}\left(k_{\rho} \rho\right) e^{j n \phi} e^{-j k_{z} z}
\end{aligned}
$$

for TE modes, where

$$
k_{z}^{2}=\sqrt{k^{2}-k_{\rho}^{2}}, \quad k_{\rho}=\frac{x_{n p}^{\prime}}{a},
$$

$a$ is the guide radius, $k$ is the free space wave number, and $x_{n p}$ and $x_{n p}^{\prime}$ are the $p$-th zeros of $J_{n}$ and $J_{n}^{\prime}$, respectively.

The aperture field can be expressed in a two-dimensional Fourier-Bessel series as

$$
\mathbf{H}^{a}=\sum_{n=0}^{\infty} \sum_{p=1}^{\infty}\left(c_{n p}^{T M} \mathbf{h}_{n p}^{T M}+c_{n p}^{T E} \mathbf{h}_{n p}^{T E}\right)
$$

where $\mathbf{h}_{n p}$ are the normalized mode vectors with

$$
\iint_{\text {cross section }}\left|h_{n p}\right|^{2} d s=1
$$

From equation (7), the Fourier expansion coefficients are found as

$$
c_{n p}=\int_{0}^{a} \int_{0}^{2 \pi} \mathbf{H}^{a}(\rho, \phi) \cdot \mathbf{h}_{n p}(\rho, \phi) \rho d \rho d \phi .
$$

For the uniform aperture field in equation (4),

$$
\mathbf{H}^{a}=\left|H^{a}\right|\left(\hat{\mathbf{a}}_{\rho} \cos \phi-\hat{\mathbf{a}}_{\phi} \sin \phi\right) .
$$




\section{Power Dissipation in a Matched Load Terminated Waveguide}

For each mode, the dissipated power in the waveguide is the dissipated power in the waveguide wall plus the dissipated power in the matched load. The dissipated power in the load equals the time average power flow through the waveguide. The total power dissipation

$$
\begin{aligned}
P_{d} & =P_{w a l l}+P_{l o a d} \\
& =\int_{0}^{2 \pi} \int_{0}^{\ell} R_{s}|H(a, \phi, z)|^{2} d \phi d z+\operatorname{Re}\left\{\int_{0}^{a} \int_{0}^{2 \pi} \mathbf{E} \times \mathbf{H}^{*} \cdot \hat{\mathbf{a}}_{z} \rho d \rho d \phi\right\}
\end{aligned}
$$

where $E$ and $H$ are the fields inside the waveguide supported by the aperture field in equation (7).

The $n p-t h$ modal electric field is

$$
E_{n p}=Z^{\omega} H_{n p}
$$

where the wave impedances are given as

$$
Z^{\omega, T_{w 1}}=\frac{E_{\rho}}{H_{\phi}}=\frac{k_{z}}{\omega \epsilon}
$$

for TM modes and

$$
Z^{\omega, T E}=-\frac{E_{\beta}}{H_{\rho}}=\frac{\omega \mu}{k_{z}}
$$

for $\mathrm{TE}$ modes. it a frequency below the cut-oir for the dominant $T E_{11}$ mode of the waveguide, ignoring the wall loss, all the waveguide modes have purely reactive input impedance. The input impedance of the matched load terminated waveguide at the aperture may be found using the transmission line theory. If the length $\ell$ of the waveguide is sufficiently long, the resistive component of the input impedance is negligible and, therefore, the time average power flow in the waveguide is negligible. 


\section{Power Dissipation in a Short-Circuited Waveguide}

In a terminated cylindrical wareguide, the magnetic field ware of the $n p-t h$ mode is

$$
H_{n p}(z)=H_{n p}^{+} e^{-\gamma_{n p} z}\left[1-\Gamma_{n p}(z)\right]
$$

where

$$
\Gamma_{n p}(z)=\frac{H_{n p}^{-}}{H_{n p}^{+}} e^{2 \gamma_{n p} z}
$$

the propagation constant

$$
\gamma=\alpha+j \beta,
$$

and $\mathrm{H}^{+}$and $\mathrm{H}^{-}$are the incident and the reflected waves, respectively. In a shortcircuited waveguide, $H^{-} / H^{+}=1$ at the short $z=\ell$. The total power lost in the waveguide section is the power dissipated on the waveguide wall plus the power dissipated on the short.

$$
\begin{aligned}
P_{d} & =P_{\text {wall }}+P_{\text {short }} \\
& =\int_{0}^{2 \pi} \int_{0}^{\ell} R_{s}|H(a, \phi, z)|^{2} d \phi d z+\int_{0}^{a} \int_{0}^{2 \pi} R_{s}|H(\rho, \phi, \ell)|^{2} \rho d \rho d \phi .
\end{aligned}
$$

At a frequency below the cut-off of the waveguide mode, the modal field evanesces rapidly and the dissipated power on the short can be ignored if the length $\ell$ is sufficiently long. 


\section{Power Dissipation in a Coaxial Transmission Line Section}

Figures 2(a) and 2(b) show the coaxial transmission line attached to a carity through a probe and a loop, respectively. The current induced on the probe is

$$
I=\frac{\partial}{\partial t} \iiint_{\text {probe }} \epsilon_{o} E d v \simeq j \omega \epsilon_{o} A E
$$

where $\mathrm{A}$ is the probe surface area, and the voltage induced on the loop is

$$
V=\frac{-\partial}{\partial t} \iint_{\text {loop }} \mu_{o} \mathbf{H} \cdot d \mathbf{s} \simeq-j \omega \mu_{o} S H
$$

where $S$ is the area of the loop. The coaxial transmission line has a diameter much less than a wavelength and thus coupling of the $T M$ and $T E$ waveguide modes will be ignored. In the coaxial line, only a $T E M$ mode will be excited with no frequency limit. For a short-circuited coaxial transmission line section, the standing wave fields are

$$
\begin{aligned}
& \mathbf{E}=\hat{\mathbf{a}}_{\rho} E_{o} \sin (k z)=\frac{\hat{\mathbf{a}}_{\rho}}{\rho} \frac{V}{\ln (a / b)} \sin (k z) \\
& \mathbf{H}=\hat{\mathbf{a}}_{\phi} \frac{E_{o}}{\eta} \cos (k z)
\end{aligned}
$$

where $\eta$ is the free space wave impedance. The voltage induced on the $E$-probe is

$$
V=Z^{\text {in }} I \text {. }
$$

The total power dissipated in the inner and outer conductor walls and on the short is

$$
\begin{aligned}
P_{d} & =P_{i c}+P_{o c}+P_{s c} \\
& =\int_{0}^{2 \pi} \int_{0}^{\ell} R_{s}\left(\left|H_{\phi}(b, \phi, z)\right|^{2}+\left|H_{\phi}(a, \phi, z)\right|^{2}\right) d \phi d z \\
& +\int_{b}^{a} \int_{0}^{2 \pi} R_{s}\left|H_{\phi}(\rho, \phi, \ell)\right|^{2} \rho d \rho d \phi .
\end{aligned}
$$

Note that, if $P_{s c}$ is ignored, the power dissipation is inversely proportional to the conductor radius of the coaxial structure for a fixed characteristic impedance $Z_{0}$. 
VI. Results

In the following, the cases of the carity to wareguide power coupling described abore are discussed with computation results. A cylindrical pillbox carity whose $T M_{010}$ resonance occurs at $352 \mathrm{MHz}$ is used in the computation. The radius and the height of the pillbox are $0.325 \mathrm{~m}$ and $0.365 \mathrm{~m}$, respectively. $100 \mathrm{KW}$ of cavity input power is used.

Transverse magnetic modes have magnetic fields closed in the transverse plane. For equation (9), results show that only the $T E_{1 p}$ modes are excited in the waveguide due to the aperture field $\mathbf{H}^{a}$. The modal power distribution of the dissipated power in the waveguide is shown in Table 1. The cumulative power dissipation along the axis of the $8 \mathrm{~cm}$ radius waveguide is shown in Figure 3. The power dissipation versus the diameter of the attached waveguide is shown in Figure 4. The waveguide is terminated with a resistive load which is assumed to be matched for all waveguide modes. For $T E_{11}$ mode, the attenuation constant of the $8 \mathrm{~cm}$ radius waveguide is $\alpha=21.8 \mathrm{Nepers} / \mathrm{m}$. This shows that the RF power at $z=30 \mathrm{~cm}$ is more than $50 d B$ below the input power. Therefore, the time average power flow through the waveguide can be ignored.

If the guide length $L>0.1 \mathrm{~m}$ and the aperture radius $r<8 \mathrm{~cm}$, from Figure 3, the power dissipations on the short and on the wall due to the reflected field become negligible. Thus, the power dissipation in a short-circuited cylindrical waveguide attached to a pillbox cavity must be similar to the results shown in Figure 3.

The power dissipation in a short-circuited $50 \Omega$ coaxial transmission line section versus the radius of outer conductor of a coaxial line is shown in Figure 5. The length of the coaxial section is $1 \lambda$ at $352 M H z$ and the characteristic impedance of the line is $50 \Omega$. The result shows that a coaxial line with greater conductor radius dissipates less, as expected. Since $T E_{11}$ can still couple to the coaxial structure, combining the above result with the power dissipation shown in Figure 4, the optimum radius of a coaxial transmission line may be found. 


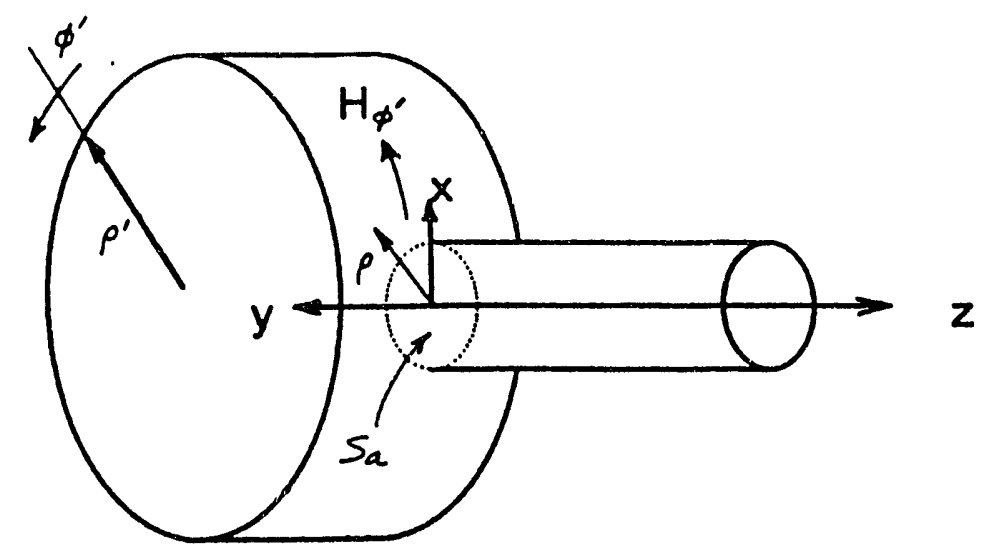

Figure 1. A cylindrical waveguide attached to a $\therefore \quad$ pillbox cavity through an aperture

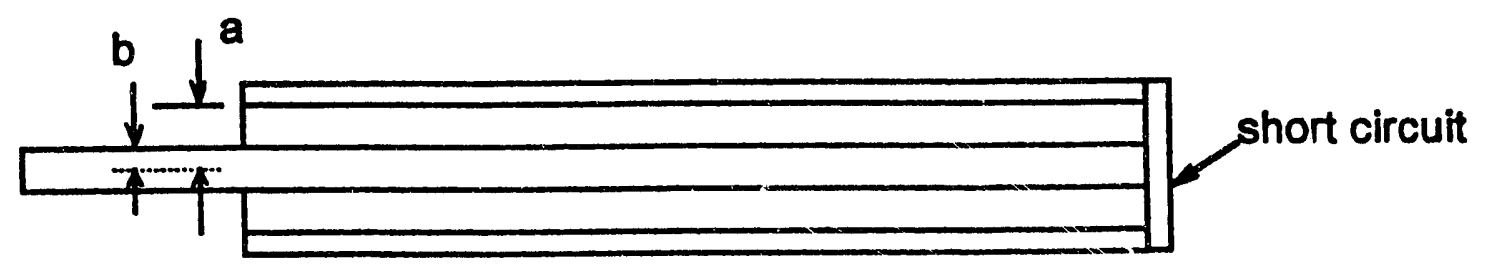

(a) Coaxial transmission line with E-probe

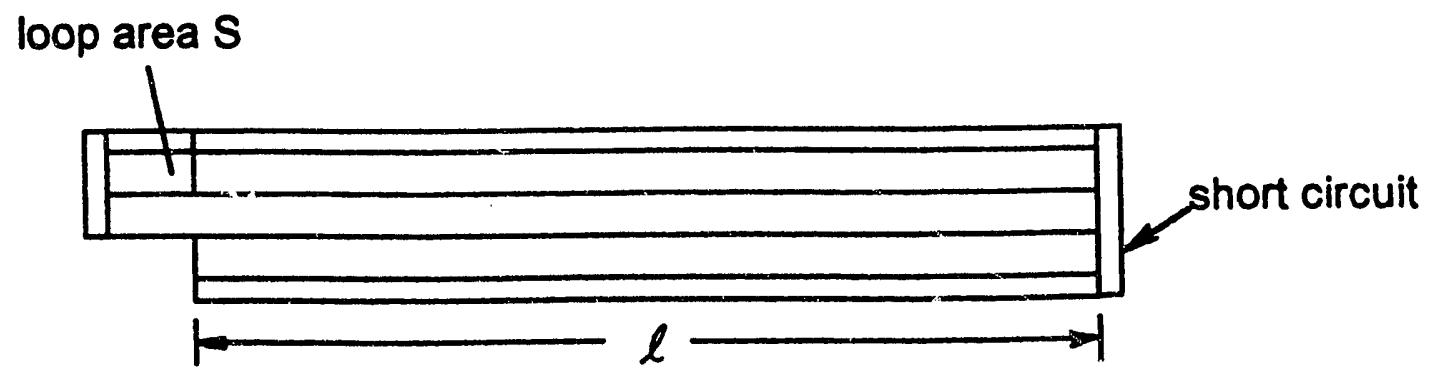

(b) Coaxial transmission line with $\mathrm{H}$-loop

Figure 2. Short-circuited coaxial transmission line sections 


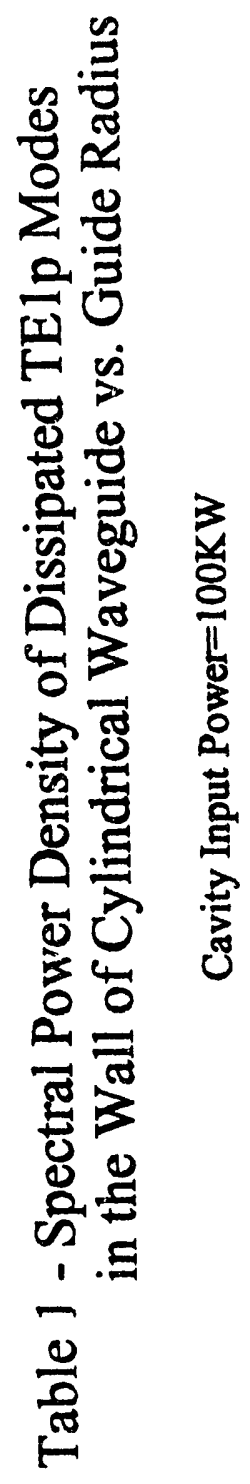

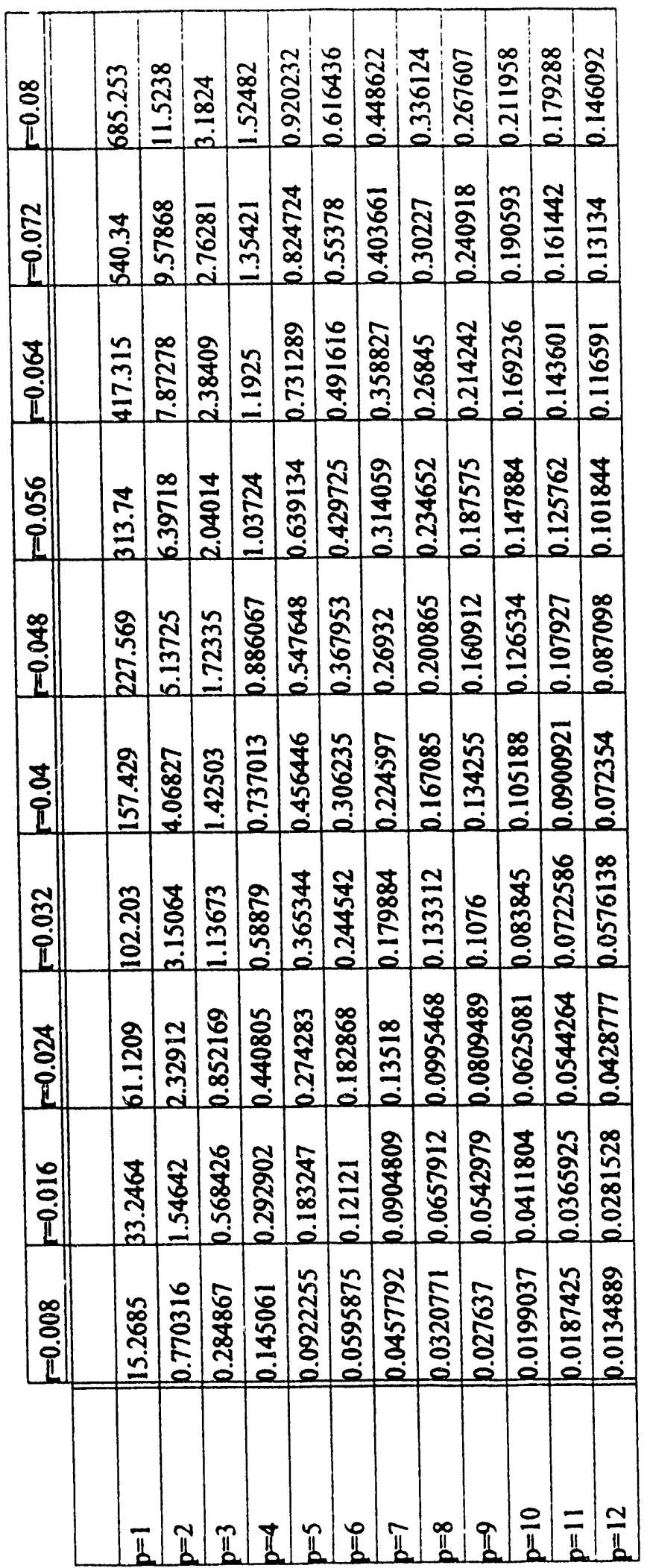




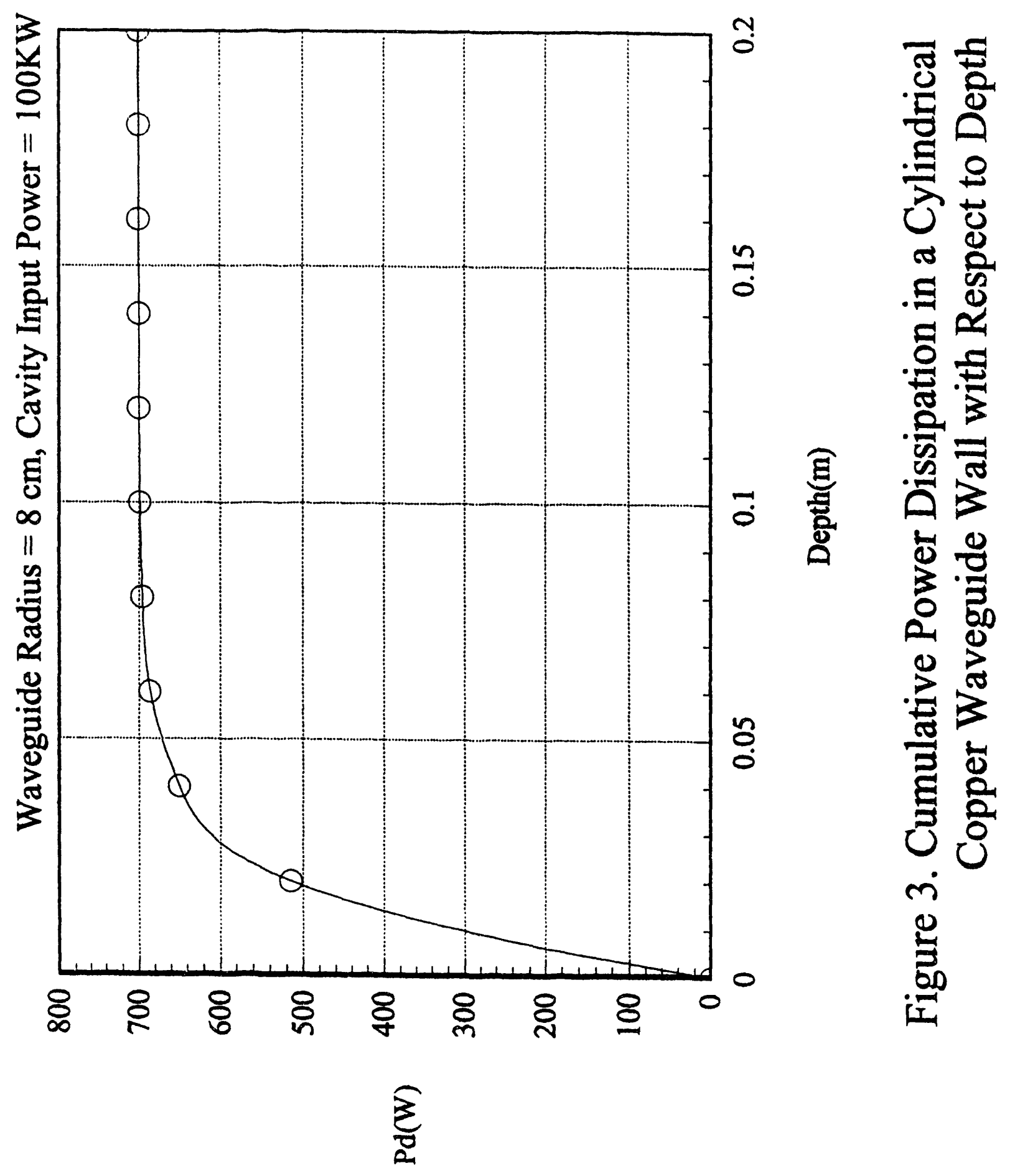



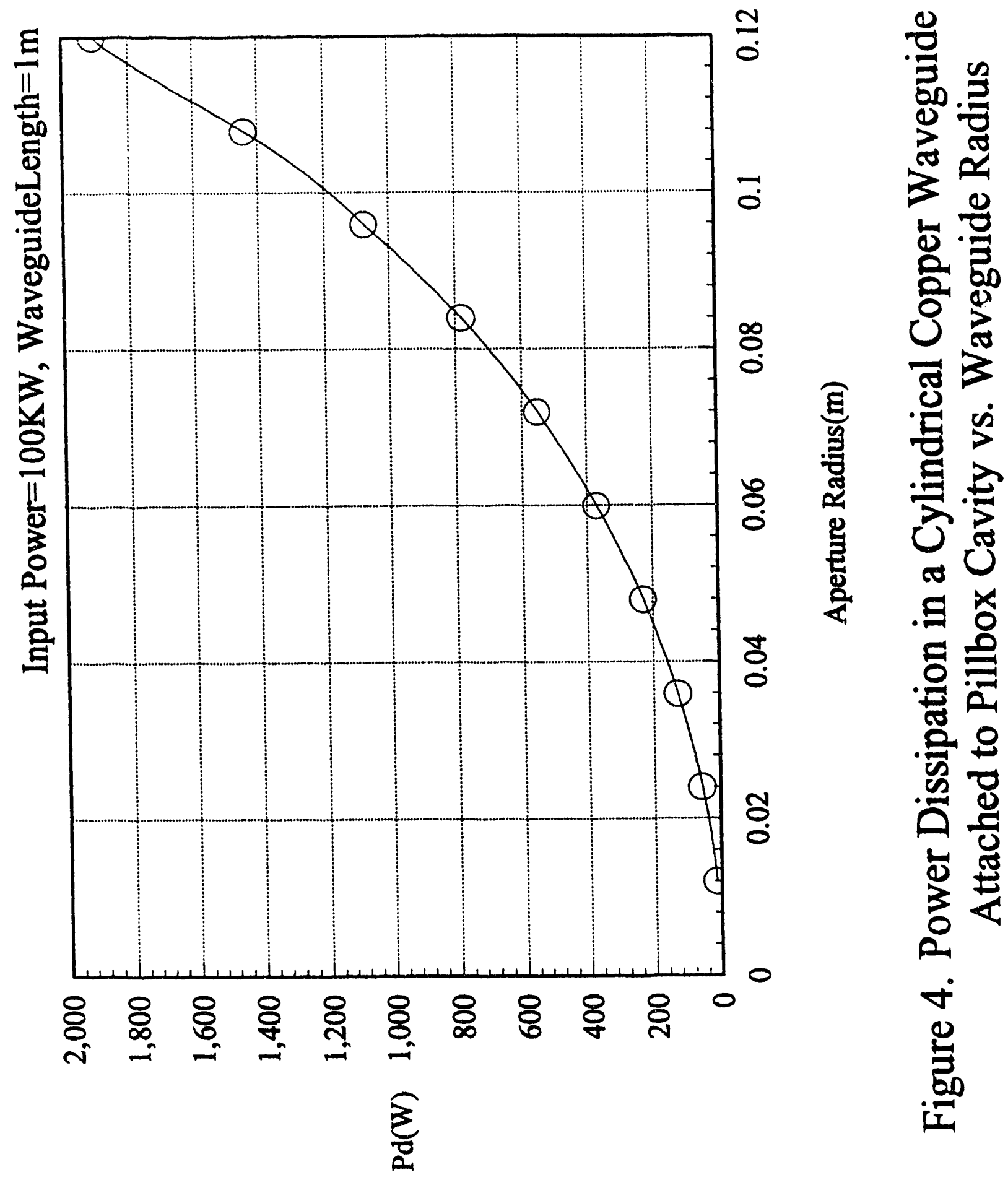


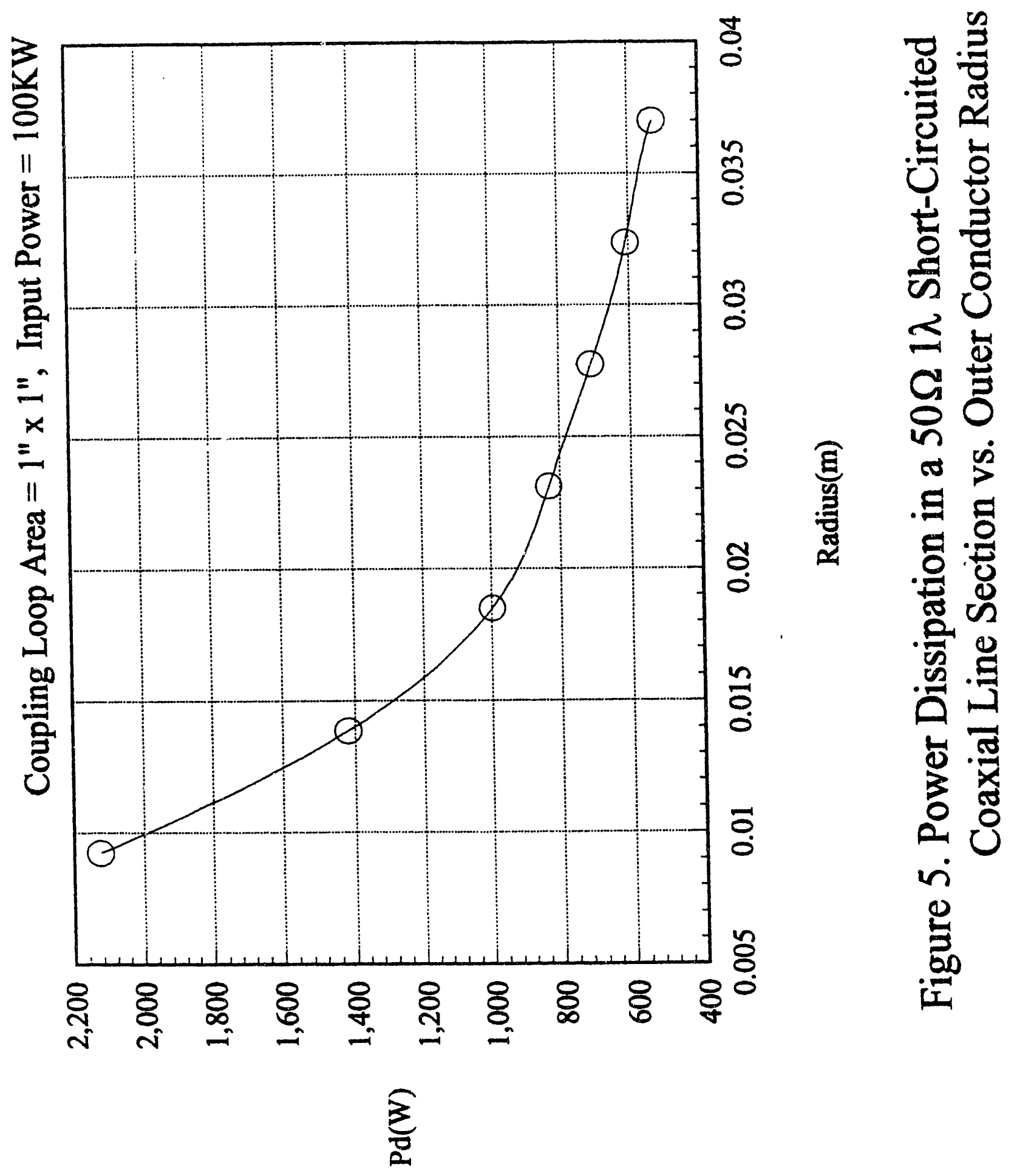


DATE FILMED $6 / 21 / 93$ 
